\title{
A Brugada syndrome revealed by Coronavirus disease (COVID 19) infection : Case report
}

\author{
Anas Hadari ${ }^{1}$, Mossab Tayane ${ }^{2}$, Laila Bendriss ${ }^{1}$ and Amine Benjelloun ${ }^{2 *}$ \\ ${ }^{1}$ Cardiology unit, Hôpital Militaire Avicenne, Marrakech, Morocco \\ ${ }^{2}$ COVID-19 Unit IV, Hôpital Militaire Avicenne, Marrakech, Morocco
}

\begin{abstract}
Since March 2020, the SARS Cov-2 outbreak is declared pandemic by the WHO. The coronavirus disease (COVID 19) is responsible for a large panel of clinical manifestations. Respiratory involvement is the most common but cardiac manifestations such as ischemia and myocarditis are not rare. We report the case of a 44-year-old patient with a history of untagged repeated syncopes admitted for a new episode associated with febrile COVID 19 infection. The ECG found a ST elevation in V1 very suggestive of Brugada syndrome. The patient was treated with hydroxychloroquine and azithromycin in addition to antipyretic therapy. This V1 elevation regressed with thermal defervescence without episode of ventricular arrhythmia. We discuss the mechanisms of Brugada syndrome and its triggers in the light of the literature.
\end{abstract}

\section{Introduction}

COVID-19 is a SARS Cov 2 coronavirus infection, that was first identified in Wuhan, China in early December 2019. It was initially described as a pneumonia of unknown cause. The viral pathogen was identified in January 2020, then it spread so rapidly that the global pandemic was declared by WHO in March 2020 [1]. In cardiology, this viral infection can trigger or reveal certain cardiovascular pathologies such as coronary syndromes, myocarditis, heart failure and others. Our observation illustrates the revelation by the coronavirus SARS Cov 2 of an unknown Brugada syndrome.

\section{Case report}

A 44-year-old patient without cardiovascular risk factors, but with a history of frequent syncopes at the age of 20 and several episodes of nocturnal palpitations without ECG or holter ECG diagnosis, was admitted to emergency room for a new episode of syncopes occurring at his workplace with atypical chest pain. People around him reported a few seconds loss of consciousness followed by a spontaneous recovery. The patient also reported a fever that appeared 5-day before with a dry cough.

On admission, the patient was conscious with a $38.2^{\circ} \mathrm{C}$ fever, a 61 bpm heart rate a $13 / 7$ blood pressure and normal respiratory parameters ( $\mathrm{SaO} 2$ at $95 \%$ ). Chest radiography and biology showed no anomalies. An electrocardiogram (ECG) showed an over-shift of the ST segment in V1 and V2 evoking a Brugada type 1 aspect without ventricular arrhythmia (Figure 1) [2,3] . Troponin dosed twice an hour apart was negative. A transthoracic doppler echocardiography was performed, showing a preserved systolic and diastolic cardiac function without morphological abnormalities. No pericardial or pleural effusion was noted. The patient was therefore admitted to isolation and tested for COVID-19 on a nasopharyngeal sample using the RT-PCR technique, which proved to be positive. The patient was then transferred to the COVID-19 Isolation Unit (UIC), under strict surveillance and monitoring followed by telemetry. The treatment included in addition to antipyretics, a dual therapy: hydroxychloroquine and azithromycin according to Pr Raoult protocol [4]. No anti-arrhythmic treatment has been initiated. With thermal defervescence the ST elevation decreased significantly, and no episode of ventricular arrhythmia occurred during his stay (Figure 2).

The patient remained in solitary confinement for 16 days before the RT-PCR negativation on two nasopharyngeal samples taken 24 hours apart. The patient was then sent to 14-day confinement. A specialized consultation was planned for the management of Brugada syndrome.

\section{Discussion}

The main impairment of COVID 19 is respiratory, with bilateral pneumonia like ground glass and condensations which can progress to an acute respiratory distress syndrome. This infection can also manifest itself in an ischemic or inflammatory cardiac involvement realizing a viral myocarditis which occurs during the cytokine storm [5]. Many cases of COVID-19 manifested with myocardial infarction and elevation of the ST segment and benefited from primary percutaneous coronary intervention. The severe inflammatory response to COVID-19 causes febrile syndrome in the vast majority of patients. Our patient presented a syncope induced by COVID fever on an unrecognized Brugada syndrome [6,7]. Fever has been shown to precipitate arrhythmia in patients with Brugada syndrome, and one study found that more than half of the study cohort experienced syncope or cardiac arrest during febrile episodes [8]. The biophysical properties of the cardiac sodium channels are altered at high temperature [9]. Although the functional decline in mutated sodium channels in patients with Brugada syndrome

*Correspondence to: Amine Benjelloun, COVID-19, Unit IV, Hôpital Militaire Avicenne, Marrakech, Morocco, Tel: 212671218550, E-mail: abenji70@gmail.com

Key words: brugada syndrome, COVID-19, ventricular arrythmia, fever

Received: May 11, 2020; Accepted: May 25, 2020; Published: June 08, 2020 


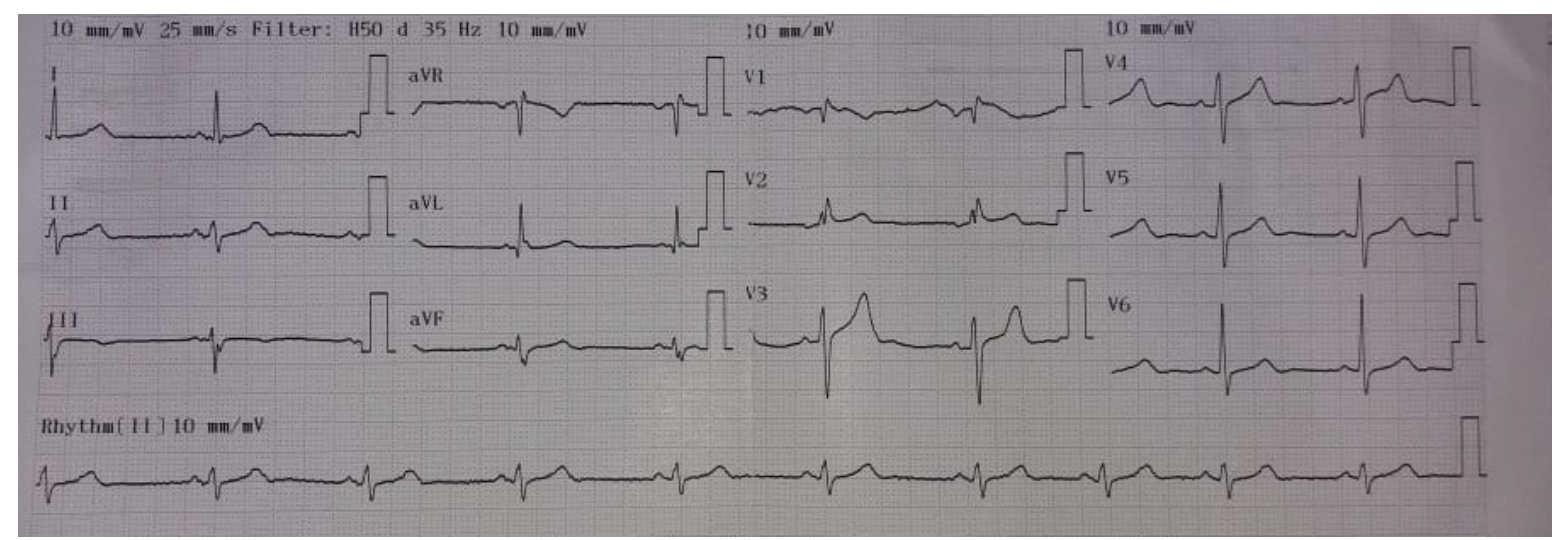

Figure 1. First ECG : Elevation of the ST segment in V1 : Brugada type 1

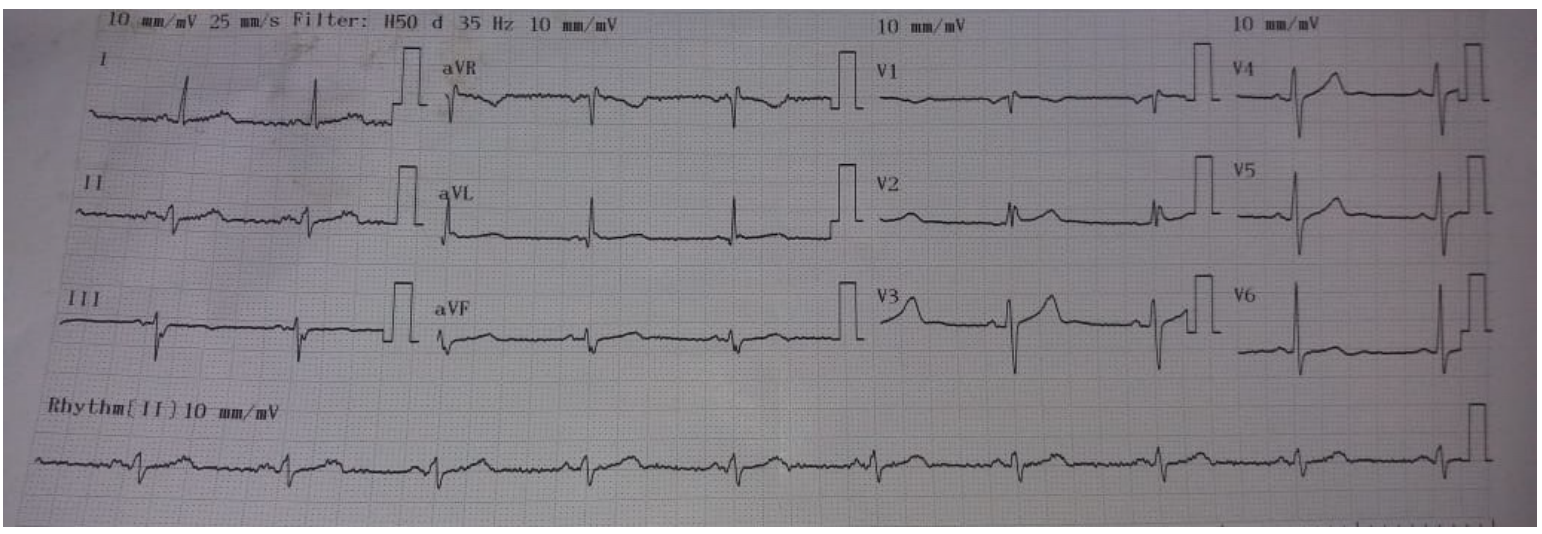

Figure 2. ECG showing normal ST segment in right sided chest lead v4R

is often minimal during temperature increases, it can result in clinical arrhythmia induced by fever [10].

The elevation of the ST segment in the right precordial leads and the electrical profiles of the Brugada type have already been described under various conditions: fever, myocarditis, metabolic disorders, certain drugs ... These aspects of the Brugada type generally disappear once the triggering event is over [11]. A Brugada-type ECG aspect presents an additional diagnostic and therapeutic challenge because it can be observed in patients with chest pain, making it difficult to differentiate with ischemia in front of an elevation of the ST segment [7].

Fever, alcohol, and drugs that block sodium channels are all potential factors for arrhythmia in patients with Brugada syndrome [12]. The most frequently used drugs for patients with SARS-CoV-2 are not contraindicated in patients with Brugada syndrome [13]. However, these patients should be subject to special attention and close monitoring, especially in case of fever, a potentially arrhythmogenic and therefore fatal factor [14].

\section{Conclusion}

We report the first case in the literature of a SARS Cov 2 coronavirus infection revealing an unrecognized Brugada syndrome. These patients should be closely monitored by telemetry or in an intensive care unit until the fever subsides, and regardless of their respiratory conditions, in order to detect early arrhythmias. In addition, there is no documented contraindication to treatment with hydroxychloroquine or azithromycin.

\section{Conflict of interest}

The authors declare having no conflict of interest related to this article.

\section{References}

1. World Health Organization. WHO Announces COVID-19 Outbreak a Pandemic?

2. Nademanee K, Veerakul G, Nimmannit S, Chaowakul V, Bhuripanyo K, et al. (1997) Arrhythmogenic marker for the sudden unexplained death syndrome in Thai men. Circulation 96: 2595-2600. [Crossref]

3. Antzelevitch C, Brugada P, Borggrefe M, Brugada J, Brugada R, et al. (2005) Brugada Syndrome: Report of the Second Consensus Conference: Endorsed by the Heart Rhythm Society and the European Heart Rhythm Association. Circulation 111: 659670. [Crossref]

4. Million M, Lagier JC, Gautret P, Colson P, Raoult D, et al. (2020) Early treatment of COVID-19 patients with hydroxychloroquine and azithromycin: A retrospective analysis of 1061 cases in Marseille, France. Travel Med Infect Dis 101738. [Crossref]

5. Li Q, Guan X, Wu P, Wang X, Zhou L et al. (2020) Early transmission dynamics in Wuhan, China, of novel coronavirus-infected pneumonia. N Engl J Med 382: 1199-1207.

6. Chang D, Saleh M, Bengo YG, Choi E, Epstein L, et al. (2020) COVID-19 infection unmasking Brugada syndrome. HeartRhythm Case Rep 6: 237-240. [Crossref]

7. Vidovich MI (2020) Transient Brugada-like ECG pattern in a patient with Coronavirus Disease 2019 (COVID-19). JACC Case Rep 32310242. [Crossref]

8. Amin AS, Meregalli PG, Bardai A (2015) Fever increases the risk for cardiac arrest in the Brugada syndrome. Ann Intern Med 149: 216-218. [Crossref]

9. Dumaine R, Towbin JA, Brugada P, Vatta M, Nesterenko DV, et al. (1999) Ionic mechanisms responsible for the electrocardiographic phenotype of the Brugada syndrome are temperature dependent. Circ Res 85: 803-809. [Crossref]

10. Keller DI , Rougier JS, Kucera JP, Benammar N, Fressart V, et al. (2005) Brugada syndrome and fever: genetic and molecular characterization of patients carrying SCN5A mutations. Cardiovasc Res 67: 510-519. [Crossref] 
11. Baranchuk A, Nguyen T, Ryu MH, Femenía F, Zareba W, et al. (2012) Brugada phenocopy: new terminology and proposed classification. Ann Noninvasive Electrocardiol 17: 299-314. [Crossref]

12. Sarquella-Brugada G, Campuzano O, Arbelo E, Brugada J, Brugada R (2016) Brugada syndrome: clinical and genetic findings. Genet Med 18: 3-12. [Crossref]
13. Postema PG, Wolpert C, Amin AS, Probst V, Borggrefe M, et al. (2009) Drugs and Brugada syndrome patients: review of the literature, recommendations, and an up-todate website. Heart Rhythm 6:1335-1341. [Crossref]

14. Michowitz Y, Milman A, Sarquella-Brugada G, Andorin A, Champagneet J, et al (2018) Fever-related arrhythmic events in the multicenter Survey on Arrhythmic Events in Brugada Syndrome. Heart Rhythm15: 1394-1401. [Crossref]

Copyright: $\odot 2020$ Hadari A. This is an open-access article distributed under the terms of the Creative Commons Attribution License, which permits unrestricted use, distribution, and reproduction in any medium, provided the original author and source are credited. 